\title{
Integration of molecular marker and doubled haploid technologies for wheat breeding in the North Africa region
}

\author{
Udupa S.M. ${ }^{1 *}$, El-Haddoury J. ${ }^{2}$, Hamza S. ${ }^{3}$, Djenadi C. ${ }^{4}$, Benbelkacem A. ${ }^{4}$, \\ Hamami R. ${ }^{5}$, Henkrar F. ${ }^{1}$, Meamiche H. ${ }^{4}$, Grana Z. ${ }^{1,2,6}$, Ghizlane D. ${ }^{7}$, Ouabbou H. ${ }^{2}$, \\ Ibriz M. ${ }^{6}$, Iraqi D. ${ }^{7}$, Slim A. ${ }^{8}$, Tsivelikas A. ${ }^{1}$, Amri A. ${ }^{1}$, Forgeois P. ${ }^{9}$, Chao S. ${ }^{10}$ \\ ${ }^{1}$ International Center for Agricultural Research in the Dry Areas (ICARDA), Rabat, Morocco \\ ${ }^{2}$ Institut National de la Recherche Agronomique (INRA), Settat, Morocco \\ ${ }^{3}$ Institut National de Recherche Agronomique de Tunis, Tunis, Tunisia \\ ${ }^{4}$ Institut National de la Recherche Agronomique d'Algérie, Algeria \\ ${ }^{5}$ Institut National de Recherche Agronomique de Tunis, Tunis, Tunisia \\ ${ }^{6}$ Ibn Tofail University, Kenitra, Morocco \\ ${ }^{7}$ Institut National de la Recherche Agronomique (INRA), Settat, Morocco \\ ${ }^{8}$ National Gene Bank of Tunisia, Tunis, Tunisia \\ ${ }^{9}$ Institut de Genech, Genech, France \\ ${ }^{10}$ USDA-ARS, Fargo, USA \\ *e-mail:S.Udupa@cgiar.org; sripada.udupa@gmail.com
}

Wheat is the major staple food crops of Morocco, Algeria and Tunisia and grown mainly under rainfed conditions. Biotic stresses such as yellow rust, leaf rust and the Hessian fly and abiotic stresses mainly drought constrains for wheat production, resulting in insufficiency in wheat production and import wheat most of the year for its domestic consumption. Developing stress tolerant varieties is one of the most efficient and economical approach to manage these stresses. However, traditional breeding approaches are in-efficient and take long time (10 25 years). As a first step and baseline information, we evaluated phenotypic and genotypic diversity of a set of improved and local landraces of bread wheat cultivars from Morocco, Algeria and Tunisia. The results revealed that many of the wheat cultivars were susceptible to prevailing biotypes of the Hessian fly and yellow rust. Therefore, the exotic wheat cultivars having various useful known genes were deployed and being used to make crosses with the North African cultivars. Integration of molecular marker and doubled haploid technologies within traditional breeding systems had enabled to select superior genotypes for traits that are difficult to select based solely on phenotype or to pyramid desirable combinations of genes into a single genetic background. The targeted crosses were made and subsequent generations were carried forward through traditional breeding systems and also in some cases through doubled haploids $(\mathrm{DH})$ to speed up development of homozygous plants. Though marker-assisted breeding (MAB) can be applied to all segregating generations, we most commonly applied to early generations, including haploids, $\mathrm{F}_{2}, \mathrm{BC}_{1} \mathrm{~F}_{1}, \mathrm{BC}_{1} \mathrm{~F}_{2}$ and the $\mathrm{F}_{1}$ of complex crosses to enrich populations with favourable genes and their combinations. $\mathrm{MAB}$ also offered the opportunity to hasten transfer of desirable alleles from un-adapted exotic genetic backgrounds into a desirable germplasm through cross-breeding. Once, the selected homozygous genotypes were validated for the selected traits under field conditions, the selected lines were seed increased for subsequent preliminary yield trials and multilocation trials. In Morocco, three bread wheat varieties namely 'Kharoba', 'Khadija' and 'Malika' were released using these tools. In Algeria and Tunisia, integration of these tools in breeding is in progress. In conclusion, application of MAB and DH technologies greatly enhanced efficiency and effectiveness of utilization of the germplasm and enhanced genetic gains in the breeding programs.

Acknowledgements: The authors are grateful to the International Treaty for Plant Genetic Resources for Food and Agriculture/FAO, the European Union, the CRP-Wheat (http:// wheat.org) and ICARDA/Morocco Collaborative Grants Program for the financial support. The views expressed herein can in no way be taken to reflect the official opinion of the European Union. 